\title{
Role of poly(ADP-ribose) polymerase in sulfur mustard toxicity
}

\author{
Malgorzata Debiak ${ }^{a}$, Kai Kehe ${ }^{b}$, Alexander Bürkle ${ }^{a, *}$ \\ a Molecular Toxicology Group. Department of Biology, Box X911, University of Konstanz, 78457 Konstanz, Germany \\ ${ }^{b}$ Bundeswehr Institute of Pharmacology and Toxicology, Neuherbergstr. 11, 80937 Munich, Germany
}

\begin{abstract}
A B S T R A C T
Sulfur mustard (SM) is a chemical warfare agent leading to severe blistering of skin and mucosal surfaces, and as a long-term effect, to an increased risk for malignancies. At the molecular level, SM acts as a bifunctional alkylating agent, leading to DNA mono-adducts and di-adducts. This review is focussed on the role of poly(ADP-ribosyl)ation in the cell and tissue responses to SM-induced damage and potential role of inhibitors of poly(ADP-ribosyl)ation as therapeutic agents for SM injury.
\end{abstract}

\author{
Keywords: \\ Sulfur mustard \\ PARP \\ DNA repair \\ Cell death \\ NAD ${ }^{+}$ \\ 3-Aminobenzamide
}

\section{Introduction}

Sulfur mustard (2,2'-dichlorodiethylsulfide; SM) is a highly toxic and mutagenic warfare agent classified as a weapon of mass destruction. The long history of SM begins with its synthesis in the 19 th century. It was recognised early on that SM is a potent blistering agent. Along with phosgene and chlorine, it started the era of chemical weapons on the battlefields of World War I. Thereafter, it entered permanently to the international military arsenal and it is still regarded a threat for modern societies (Kehe and Szinicz, 2005). While the production of SM does not require specialised technology, the danger of terrorist attack against the civilian population is considerable. On the other hand, apart from the purposeful attack, there is a risk of accidental exposure to SM connected with inappropriate disposal of old depots (Geraci, 2008). Paradoxically, SM has found applications in medicine. The original compound has been in use for some time for the treatment of psoriasis (Janouchek et al., 1987). During World War II, a military research group investigated the effects of SM and its nitrogen relatives on rapidly dividing cells. These efforts resulted in medical applications of alkylating mustard compounds. The first therapy of leukaemia with nitrogen mustard was highly effective (Goodman et al., 1984). Thus, a number of sulfur and nitrogen mustard derivatives such as cyclophosphamide, chlo-

\footnotetext{
* Corresponding author. Tel.: +49 7531884035 ; fax: +49 7531884033 .

E-mail address: alexander.buerkle@uni-konstanz,de (A. Bürkle).
}

rambucil and melphalan are currently used as common anti-cancer drugs. In addition, recognition of SM as an immunosuppressant compound prompted studies on chemical immunosuppression. These studies finally paved the way for organ transplantation (Patel, 2006).

As soon as SM was first used as a warfare agent, research aimed at the development of an effective antidote was launched. Despite extensive investigation, the expected progress has been rather disappointing so far. Different clinical approaches including application of antioxidants, scavengers, anti-inflammatory drugs and protease inhibitors have been tested for treatment of the acute injuries but none of them gave full protection against formation of delayed pathology (Casillas et al., 2000). This review is focussed on the role of poly(ADP-ribosyl)ation in the cell and tissue responses to SM-induced damage. Poly(ADP-ribosyl)ation is one of the most drastic post-translational protein modifications and plays a key role in a wide range of biological processes, including DNA repair, transcriptional regulation and cell death signalling. Early studies with first-generation inhibitors of poly(ADP-ribosyl)ation have already revealed some promising therapeutic potential in SM skin injury (Cowan et al., 2003). The availability of new, more effective inhibitors brings it back to the centre of interest.

\section{Cellular targets of sulfur mustard}

Exposure to $S M$ is seldom fatal but leads to severe injuries, demanding long-term medical support. The most affected organs 
are skin, the eyes and the respiratory tract, i.e. tissues coming in direct contact with the toxic liquid or vapour. SM is a powerful blistering agent. The formation of thin-walled blisters is preceded by an asymptomatic latent period of several hours, followed by itching, pain and erythema. Cytotoxicity is primarily apparent in basal keratinocytes but with time cellular degeneration can be observed within all layers of skin. The separation of epidermis and dermis, which underlies blister formation, is accompanied by local inflammation. Progression of the lesions and the degree of blistering and necrosis is depending on dose of SM delivered (Evison et al., 2002; Ruff and Dillman, 2007).

SM is a bifunctional alkylating agent, reacting readily with most cellular macromolecules including proteins and nucleic acids. The reaction with DNA leads to rapid formation of mono- and diadducts. The majority of DNA alkylation consists of adducts at the $\mathrm{N}-7$ position of guanine and $\mathrm{N}-3$ position of adenine, accounting for 61 and $16 \%$ of total alkylations, respectively (Ludlum et al., 1994). DNA di-adducts, on the other hand, represent about $15 \%$ of SM-induced DNA lesions and are formed by reaction of one SM molecule with N-7 positions of two guanines. Di-adducts occur within the same or complementary DNA strands, yielding intra- and interstrand cross-links, respectively. The formation of intrastrand cross-links is favoured (Ludlum and Papirmeister, 1986).

SM is a relatively poor inducer of DNA strand breaks. Mol et al. quantified the number of DNA single strand breaks and/or alkali-labile sites in cultured human epidermal keratinocytes by using the alkaline elution method. The induction of DNA singlestrand breaks, measured immediately after $30 \mathrm{~min}$ exposure, was dose-dependent. Assuming a linear dose-effect relationship, it was calculated that $1 \mu \mathrm{M}$ SM induces approximately 0.05 breaks $/ 10^{9} \mathrm{Da}$ of DNA, which corresponds to 0.2 Gy of ionizing radiation. For comparison, under the same conditions, the number of interstrand cross-links was estimated to be 0.12 cross-links $/ 10^{9} \mathrm{Da}$ of DNA (Mol et al., 1993). Further incorporation of DNA strand breaks might be initiated during the repair process of DNA alkylations. The dependence of strand breaks formation on apurinic endonuclease after SM exposure was shown (Papirmeister et al., 1985). These DNA strand breaks are in turn recognised by PARP-1 (see below).

Although the DNA-damaging properties of SM are essential for its toxicity, the reactivity with other macromolecules should not be neglected. It was found that cytoskeletal changes, such as loss of stress fibres and rounding of the cells, proceed in endothelial cells after SM treatment even in absence of the cell nucleus, i.e. in cytoplasts. However, in contrast to endothelial cells, endothelial cytoplasts did not show the hallmarks of apoptosis such as caspase3 activation and externalisation of phosphatidylserine (Lodhi et al., 2001). Thus, DNA alkylation seems to be responsible for triggering of apoptosis but not for all of the cytotoxic effects of SM.

The interaction of SM with cellular antioxidants system deserves special interest. SM reacts readily with cellular thiols such as glutathione (GSH). The depletion of intracellular levels of GSH was reported in cell culture systems and in vivo models (Vijayaraghavan et al., 1991; Pant et al., 2000; Kumar et al., 2001). Furthermore, the depletion of key antioxidant enzymes such as superoxide dismutase, catalase and glutathione peroxidase was observed (Husain et al., 1996; Jafari, 2007). The exhaustion of the cellular antioxidant system results in accumulation of endogenous reactive oxygen species (ROS) and subsequent secondary damage of macromolecules, including lipid peroxidation and DNA damage (Paromov et al., 2007). The reaction with DNA would lead to further activation of PARP-1.

\section{Poly(ADP-ribose) polymerase-1: a protein for al occasions}

Poly(ADP-ribosyl)ation is a post-translational protein modification induced by genotoxic treatment in multicellular organisms. The reaction is catalysed by the family of poly(ADP-ribose) polymerases (PARPS) encoded in humans by more than a dozen different genes. Two family members, i.e. PARP -1 and PARP-2, have been shown to be activated by DNA strand breaks. By using NAD ${ }^{+}$as a substrate, PARPs build up homopolymeric branched chains of ADP-ribose [poly(ADP-ribose); PAR] of different length that are covalently bound to "acceptor" proteins at glutamic and aspartic acid or lysine residues. PAR may comprise up to 200 ADP-ribose units, coupled with each other via unique ribose $\left(1^{\prime \prime} \rightarrow 2^{\prime}\right)$ ribose bonds, and displays multiple branching points each $20-50$ residues resulting from the formation of ribose $\left(1^{\prime \prime \prime} \rightarrow 2^{\prime \prime}\right)$ ribose linkages. Apart from the covalent modification of acceptor proteins, PAR has also been shown to interact non-covalently with proteins. A specific PAR-binding motif has been identified in wide range of proteins involved in DNA repair and cell cycle checkpoints (Pleschke et al., 2000). The non-covalent PAR interaction is dependent on polymer chain length and the nature of the acceptor protein. For example, both short and long polymers can interact with the tumour suppressor protein p53, while under the same experimental conditions the nucleotide excision repair protein XPA binds exclusively long chains (Fahrer et al., 2007)

Most attention has been focussed on PARP-1 [EC 2.4.2.30], which is the best-characterised family member and also displays the highest level of enzymatic activity. PARP-1 is a constitutively expressed 113-kDa nuclear protein. In the absence of DNA damage, it has a very low basal activity but becomes rapidly activated by over 100-fold in presence of DNA strand breaks. PARP-1 is able to bind directly to single as well as to double-strand breaks. However, several groups have provided evidence that PARP-1 also recognises structural changes in DNA other than strand breaks, including distortions in DNA helical backbone and three and four-way junctions. Upon treatment of cells with DNA-damaging agents, $90 \%$ of cellular PAR is synthesized by PARP-1. The major "acceptor" protein (i.e. protein undergoing covalent modification with PAR) is PARP-1 itself, in the context of the so-called automodification reaction, but many other acceptor proteins have been described, including $\mathrm{p} 53$, both subunits of NF- $K B$, histones, DNA topoisomerases and the catalytic subunit of DNA-dependent protein kinase (DNA-PK). Due to the high negative charge of PAR, this modification significantly alters the physical and biochemical properties of the modified proteins including their DNA-binding affinity, and it is likely that such alteration will have a regulatory function concerning the interaction with other proteins.

A complete description of the multifaceted biological functions of PAR is beyond the scope of this review. Instead we focus here on those aspects that have a putative impact on the processing of SM lesions, i.e. the regulation of DNA repair, cell death and transcription. The biological function of PARP-1 that is established best is the contribution in the maintaining of survival and genomic integrity under genotoxic stress. PARP- 1 becomes activated within minutes upon treatment with DNA damaging agents and promotes survival of cells. Cells and mice lacking functional PARP-1 are hypersensitive to a number of DNA-damaging agents such as ionizing radiation, alkylating agents or oxidants. The protective action of PARP-1 was initially ascribed to regulation of base-excision repair pathway (BER). The current view, however, assumes involvement of PARP-1 at different stages of strand break repair: recognition of damage, local relaxation of the chromatin by direct modification of the histones and finally the assembly of repair protein complex by noncovalent interaction with PAR 
Alternatively, PARP-1 has been viewed to act as a switch between long and short-patch BER pathway, depending on the availability of ATP.

Recently, it has been shown that PARP-1 plays a role beyond BER. PARP-1 is implicated in double-strand break repair by at least two different pathways. Upon binding to double-strand breaks PARP-1 initiates the so-called backup non-homologous end joining pathway (B-NHEJ). B-NHEJ operates independently of classic NHEJ but is dependent on XRCC1/ligase III (Rosidi et al., 2008; Wu et al., 2008). Furthermore, a link between PARP-1 and the "Ataxia-telangiectasia mutated" (ATM) protein was established. These proteins have been known for a long time to operate in different, independent DNA strand-break repair pathways. Recent studies, however, revealed that ATM noncovalently interacts with PAR. Moreover, the recruitment of Mre11 and NBS- 1 to the damage site and the ATM response to double-strand breaks is delayed by PARP-1 inhibition. Altogether the data suggest an upstream role of PAR in ATM regulation (Haince et al., 2007, 2008).

PARP-1 has implications in apoptotic and necrotic cell death pathways. The involvement of PAR in an intrinsic apoptotic pathway was described by the Dawson group. Upon treatment with the alkylating agent MNNG, PARP-1 becomes activated and undergoes automodification. The enzymatic degradation of polymers produces free PAR chains, which act as a death signal after translocation to mitochondria, by inducing AIF release and caspase-independent apoptosis. In turn, the specific cleavage of PARP-1 by caspase-3/7 and generation of 89 and $24 \mathrm{kDa}$ fragments have been extensively used as a biochemical marker of caspase-dependent apoptosis. Caspase-mediated cleavage of PARP-1 leads to loss of its catalytic activity and inactivation of its pro-survival function but preserves the depletion of $\mathrm{NAD}^{+}$that would ensue as a result of sustained PARP-1 activity stimulated by large numbers of strand breaks. The over-activation of PARP- 1 and consequent NAD ${ }^{+}$crisis leading to massive necrotic cell death has been demonstrated to play a major role in a wide variety of pathophysiological conditions, including ischemia-reperfusion damage and a wide range of inflammatory conditions. This scenario has also implications for SM skin injury.

Interesting results concerning PARP-1 function have been obtained using the Parp1 knockout mice model. Surprisingly, animals were resistant to multiple inflammatory disorders including endotoxin-induced septic shock. The typical up-regulation of a subset of inflammatory mediators mediated by NF- $\mathrm{kB}$ gene expression, such as TNF- $\alpha$, IFN $\gamma$ and iNOS, was impaired in Parp $1^{-1-}$ animals. It has been shown that PARP-1 physically interacts with NF- $K B$ and is indispensable for its transcriptional activation. Remarkably, there is a debate if NF- $\mathrm{KB}$ activation is dependent on PARP-1 enzymatic activity or just on PARP-1 protein expression. In any event, regulation of transcription by PARP-1 is not limited to NF-KB. Indeed, numerous physical and functional interactions with stressinducible transcription factors including AP-1 and HIF-1 $\alpha$ (Elser et al., 2008) have been described.

\section{Poly(ADP-ribose) in sulfur mustard injury}

SM exposure is connected with induction of DNA strand breaks by three possible mechanisms: (i) direct reaction of the compound with DNA; (ii) strand breaks as intermediates during the process of repair of DNA alkylation; or (iii) secondary increase in cellular ROS level. PARP-1 is a first-line protein involved in the cellular response to DNA strand breaks and becomes immediately activated upon treatment with genotoxic agents such as ionizing radiation or alkylating agents. The activation of PARP-1 in the processing of SM-induced damage is, therefore, not surprising.
The first data connecting PARP-1 with SM exposure were reported over two decades ago by Papirmeister et al. (1985). In their very attractive biochemical hypothesis they linked early DNAdamaging events with late pathology of the skin. The proposed mechanism assumed that the massive induction of DNA strand breaks in enzymatic processing of SM DNA adducts would lead to activation of PARP- 1 and consequent depletion of cellular NAD ${ }^{+}$. This in turn would result in a collapse of ATP production, followed by protease release and blister formation. The model was strongly supported by in vitro and in vivo experiments. The direct induction of DNA strand breaks upon SM exposure was not observed. However, the DNA fragmentation appeared if SM-treated DNA was incubated with recombinant bacterial apurinic endonuclease, consistent with a role of strand breaks as DNA repair intermediates. The $\mathrm{NAD}^{+}$level and pathological changes were followed in mice grafted with human skin as a model system. SM induced a time and dose-dependent decrease of $\mathrm{NAD}^{+}$concentration. The reduction was marked already by $2 \mathrm{~h}$ after treatment and reached a maximum $2 \mathrm{~h}$ later. The depletion was correlated with the severity of skin injury. Mild injury was associated with little initial decrease in $\mathrm{NAD}^{+}$level and with recovery back to control levels $18 \mathrm{~h}$ after treatment. The decrease in $\mathrm{NAD}^{+}$level below $25 \%$ of control was irreversible and correlated with severe injury. The $\mathrm{NAD}^{+}$depletion could be prevented by pre-treatment with the PARP inhibitor 3-aminobenzamide.

PARP-1 activation and severe NAD ${ }^{+}$depletion have independently been confirmed in different cell systems including primary leukocytes (Meier et al., 1987), keratinocytes and endothelial cells (Mol et al., 1989; Hinshaw et al., 1999) and also in animal models (Yourick et al., 1991; Zhang et al., 1995). In all cell culture models, SM treatment induced a time and dose-dependent decrease in $\mathrm{NAD}^{+}$level. The depletion was observed up to $2 \mathrm{~h}$ post-treatment time. The degree of $\mathrm{NAD}^{+}$loss was correlated with the SM dose applied. In addition to determinations of NAD ${ }^{+}$level, Hinshaw et al. measured PARP-1 activity in permeabilised cells by incorporation of radioactive-labelled NAD ${ }^{+}$. PARP-1 activity in keratinocytes and endothelial cells displayed a mirror image to $\mathrm{NAD}^{+}$concentration. PARP-1 activation was observed $2 \mathrm{~h}$ after treatment with $500 \mu \mathrm{M}$ SM and persisted up to $5 \mathrm{~h}$ after treatment (Hinshaw et al., 1999).

Based on the above, one may expect that if severe $\mathrm{NAD}^{+}$ consumption could be prevented this would protect against cytotoxicity of SM. The availability of cell-permeable PARP-1 inhibitors made it possible to test this hypothesis. Nevertheless, the results obtained in cell culture models appeared to be rather disappointing. While the cellular $\mathrm{NAD}^{+}$pool could indeed be preserved by treatment of cells with PARP-1 inhibitor 3-aminobenzamide (Papirmeister et al., 1985; Meier et al., 1987), this did not have any effect on total cell survival. Instead, PARP-1 inhibition favoured induction of apoptosis over necrosis. Inhibition of PARP-1 resulted in increased caspase activation, PARP-1 apoptotic cleavage and DNA laddering (Meier et al., 2000; Kehe et al., 2007; Steinritz et al., 2007). It should be noted that a protective effect of the PARP inhibitors 3-aminobenzamide or niacinamide against cell death caused by SM was shown for human lymphocytes (Meier and Johnson, 1992; Meier, 1996). In both reports, however, cytotoxicity was measured by dye exclusion. By this method only necrotic cells are scored as dead cells. The results, therefore, did not contradict other studies but are in agreement with the notion that PARP- 1 inhibition can rescue cells from necrotic cell death. It has been suggested that a switch between apoptosis and necrosis might be dependent on cellular ATP content. Apoptosis is energy-dependent, whereas necrosis is not, and can be occur without ATP supply. According to this view, activated PARP-1 would use up the NAD ${ }^{+}$pool resulting in impairment of ATP synthesis. However, this assumption does not seem to be realistic in the case of SM treatment. ATP concentration 


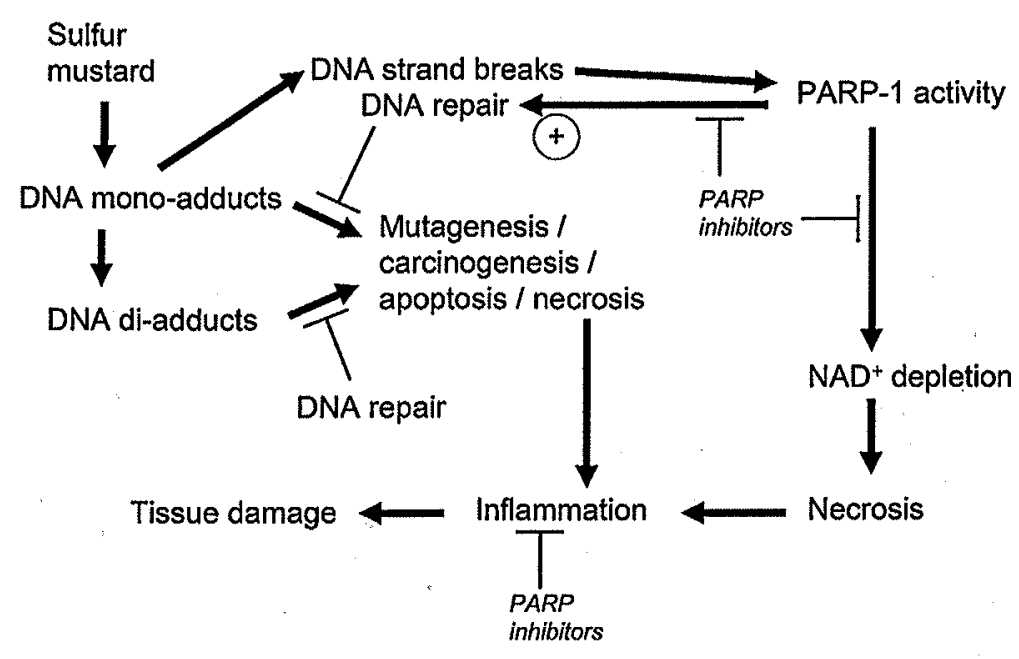

Fig. 1. Schematic of the role of PARP-1 in sulfur mustard tissue injury. For details see text.

decreased significantly in keratinocytes but only after high-dose treatment with $1 \mathrm{mM}$ SM. Lower-dose exposure did not have an impact on ATP levels despite affecting cell viability (Hinshaw et al., 1999; Kehe et al., 2007).

At this point it is worth noting that the "classical" effect of PARP inhibitors applied to proliferating cells exposed to low doses of monofunctional alkylating agents or ionizing radiation is the enhancement of cell killing, which can be explained by blockage of base-excision repair and therefore accumulation of DNA damage to lethal levels (Bürkle, 2005). The fact that such phenomenon has not been reported for (low-dose) SM treatment could mean either that base-excision repair is not of functional importance for the removal of SM-induced DNA damage or that the effect on BER is covered up by other effects of PARP inhibition such as changes in $\mathrm{NAD}^{+}$metabolism as discussed above. Presently available data do not allow distinguishing between these possibilities.

While the cytoprotective effects of PARP-1 inhibition in vitro were of limited magnitude, work on animal models revealed that it might have therapeutic value in the treatment of skin injuries. Pre-treatment with the PARP inhibitor niacinamide reduced microvesicle formation by $50 \%$ in hairless guinea pigs cutaneously exposed to SM (Yourick et al., 1991). It should be noted, however, that niacinamide may have a dual function, i.e. it also serves as a precursor to $\mathrm{NAD}^{+}$, which can render interpretation of the biological effects difficult. On the other hand, PARP inhibitors also emerged as potential therapeutic candidates in the battle drug-screening program initiated by US Army Medical Research Institute of Chemical Defence. The study included nearly $400 \mathrm{com}-$ pounds tested in the mouse ear model for skin injury. Among 19 drugs that revealed reduced histopathological changes by $>50 \%$, three different compounds were recognised as PARP inhibitors, i.e. 3-(4'-bromophenyl)ureidobenzamide, benzoylene urea and 4amino-1-naphtol hydrochloride (Cowan et al., 2003). There are two possible explanations for the protective effect of PARP-1 inhibitors in vivo. First the reduction of necrotic cell death would result in decrease of protease activity and inflammation caused by uncontrolled cell disruption. Second, the direct interference with inflammatory signalling could mitigate secondary tissue damage resulting from inflammation.

The involvement of PARP-1 in cell death regulation after SM exposure attracted most attention of researchers. The other possible consequences of PARP- 1 activation, including its primarily role in the DNA repair process, were hardly investigated. A set of stud- ies were designed to reveal the action of PARP-1 in DNA strand break repair (Bhat et al., 2000, 2006a,b). Primary keratinocytes displayed rapid phosphorylation and activation of DNA ligase 1 after SM treatment. This process was dependent on DNA-PK activity. Interestingly, the half-life of activated DNA ligase $I$ increased in the presence of 3-aminobenzamide from 1.3 to $4.3 \mathrm{~h}$. Nevertheless, the experimental system used by the authors does not provide direct evidence for the involvement of PARP-1 in SM DNA damage repair. Furthermore, comparisons were made between primary normal human keratinocytes and immortalised stably transfected cells with an inducible PARP-1 anti-sense construct. When compared to primary wild-type cells, PARP-1 deficient cells displayed impaired induction of DNA ligase I phosphorylation by SM. However, one has to keep in mind that the basal level of specific activity of ligase I in these cells was five times higher than in primary PARP-1 wild type cells. Furthermore, it was shown that the DNA repair rate in inducible PARP-1-deficient cells was about 60-70\% lower than in primary keratinocytes. On the other hand, the repair rate in induced and uninduced antisense PARP-1 cells did not differ. The differences in repair reaction velocity were apparently resulting from cell type specificity and not PARP -1 status. Anyway, the fast activation of PARP-1 indicates that the enzyme is able to recognise DNA damage induced by SM. The possible involvement of PARP-1 in facilitating DNA repair is definitively worth of more detailed investigation.

\section{Perspectives}

The current renaissance of PARP- 1 inhibitors in cancer chemotherapy, either in combination with alkylating agents (Thomas et al., 2007) or as a monotherapy against tumours that are deficient in homologous recombination (Bryant et al., 2005; Farmer et al., 2005) opens new avenues for treatment of SM injury. Initial studies with PAPR-1 inhibitors have already revealed a potential in management of SM skin injury. There is now a new generation of inhibitors available, which is over 100 times more potent than the classical ones, has improved pharmacokinetic properties and higher specificity. Several PARP inhibitors are undergoing clinical trials as drugs for cancer treatment and their pharmacodynamic properties are intensely monitored. If approval for medical use were obtained it could make available a "ready to use" drug that might soon be applied in SM therapy (Zaremba and Curtin, 2007).

There is, however, one important point to consider: SM is a known mutagen and carcinogen. In this context, inhibition of PARP- 
1 thight act as double-edged sword (Fig. 1). We do know that PARP-1 is activated upon SM-induced DNA damage. Its participation in DNA repair processes upon SM exposure is so far not clear. PARP-1 is a key player in repair of DNA damage induced by simple alkylating agents and ionizing radiation. PARP- 1 inhibition in combination with alkylating agent treatment leads to increased genomic instability and chromosome damage (Bürkle, 2005). The influence of PARP-1 inhibition on SM-induced mutagenesis should therefore beinvestigated in order to assess the safety of PARP inhibitors as potential therapeutics.

Finally, the role of PARP-1 in regulation of transcription, in particular in the expression of inflammatory cytokines, is still an under-researched field. Keratinocytes have been shown to respond to $S M$ exposure with production of inflammatory cytokines including IL-1, IL-6, IL-8 and TNF- $\alpha$. Cytokine production was blocked by inhibition of p38 kinase (Dillman et al., 2004). Parallel to MAPK activation, keratinocytes also activate the canonical NF- $\mathrm{KB}$ pathway (Rebholz et al., 2008). PARP-1, on the other hand, is an important coactivator of NF-KB in lipopolysaccharide-induced transcription of genes encoding inflammatory cytokines. Therefore, the functional in terplay between PARP-1 and NF- $\mathrm{KB}$ in SM-induced inflammation appears to be another promising aspect of future research on PARP inhibitors as a potential treatment for SM injury.

\section{Conflict of interest statement}

The authors declare they have no conflict of interest.

\section{Acknowledgement}

The authors gratefully acknowledge support through a project grant by the Bundeswehr (U2.3 E/UR3G/4G121/5A805).

\section{References}

Bhat, K.R., Benton, B.J., Ray, R,, 2006a, DNA ligase 1 is an in vivo substrate of DNAdependent protein kinase and is activated by phosphorylation in response to DNA double-strand breaks. Biochemistry 45, 6522-6528.

Bhat, K.R., Benton, B.J., Ray, R., 2006b. Poly (ADP-ribose) polymerase (PARP) is essential for sulfur mustard-induced DNA damage repair, but has no role in DNA ligase activation. J. Appl. Toxicol. 26, 452-457.

Bhat, K.R., Benton, B.J., Rosenthal, D.S., Smulson, M.E., Ray, R., 2000. Role of poly(ADPribose) polymerase (PARP) in DNA repair in sulfur mustard exposed norma human epidermal keratinocytes (NHEK). J. Appl. Toxicol. 20 (Suppl. 1) S13-S17.

Bryant, H.E., Schultz N., Thomas, H.D. Parker, KM Flower D Lopez, E., Kyle, S, Meuth, M., Curtin, N.J., Helleday, T, 2005. Specific killing of BRCA2-deficient tumours with inhibitors of poly(ADP-ribose) polymerase. Nature 434, 913-917.

Bürkle, A., 2005. Poly(ADP-ribose). The most elaborate metabolite of NAD+. FEBS J. $272,4576-4589$

Casillas, R.P., Kiser, R.C., Truxall, J.A., Singer, A.W., Shumaker, S.M., Niemuth, N.A., Ricketts, K.M. Mitcheltree, L.W. Castrejon, LR, Blank, J.A, 2000. Therapeutic approaches to dermatotoxicity by sulfur mustard. 1 . Modulaton of sulfur mustard-induced cutaneous injury in the mouse ear vesicant model. J. Appl. Toxicol. 20 (Suppl. 1), S145-S151.

Cowan, F.M., Broomfield,C.A.,Lenz, D.E.,Smith, W.J., 2003, Putative role of proteolysis and inflammatory response in the toxicity of nerve and blister chemical warfare agents: implications for multi-threat medical countermeasures. J. Appl. Toxicol. 23, 177-186.

Dillman III, J.F., McGary, K.L., Schlager, J.J., 2004. An inhibitor of p38 MAP kinase downregulates cytokine release induced by sulfur mustard exposure in human epidermal keratinocytes. Toxicol, In Vitro 18, 593-599.

Elser, M., Borsig, L., Hassa, P.O., Erener, S., Messner, S., Valovka, T, Keller, S., Gassmann M. Hottiger, M.O., 2008. Poly(ADP-ribose) polymerase 1 promotes tumor cel survival by coactivating hypoxia-inducible factor-1-dependent gene expression. Mol. Cancer Res. 6, 282-290.

Evison, D., Hinsley, D., Rice, P., 2002. Chemical weapons. BM] 324, 332-335.

Fahrer, J., Kranaster, R., Altmeyer, M., Marx, A., Bürkle, A., 2007. Quantitative analysis of the binding affinity of poly(ADP-ribose) to specific binding proteins as a function of chain length. Nucleic Acids Res, 35, e143.

Farmer, H. McCabe, N Lord, C.J. Tutt, A.N. Johnson, D.A. Richardson, T.B. Santarosa, M. Dillon, K. ., Hickson, 1., Knights, C., Martin, N.M., Jackson, S.P., Smith, G.C. Ashworth, A., 2005. Targeting the DNA repair defect in BRCA mutant cells as a therapeutic strategy. Nature 434, 917--921.
Geraci, M.J., 2008. Mustard gas: imminent danger or eminent threat? (February) (CE). Ann. Pharmacother. 42, 237-246.

Goodman, L.S., Wintrobe, M.M., Dameshek, W., Goodman, M.J. Gilman, A., McLennan, M.T. 1984. Landmark article September 21, 1946 Nitrogen mustard therapy. Use of methyl-bis(beta-chloroethyl)amine hydrochloride and tris(beta-chloroethyl)amine hydrochloride for Hodgkin's disease, lymphosarcoma, leukemia and certain allied and miscellaneous disorders. JAMA 251 2255-2261.

Haince, J.F, Kozlov, S., Dawson, V.L., Dawson, T.M., Hendzel, M.J., Lavin, M.F., Poirier G.G., 2007. Ataxia telangiectasia mutated (ATM) signaling network is modulated by a novel poly(ADP-ribose)-dependent pathway in the early response to DNAdamaging agents. J. Biol. Chem. 282, 16441-16453.

Haince, J.F., McDonald, D., Rodrigue, A, Dery, U., Masson, J.Y., Hendzel, M.J., Poirier G.G., 2008. PARP1-dependent kinetics of recruitment of MRE11 and NBS1 proteins to multiple DNA damage sites. J. Biol. Chem. 283, 1197-1208.

Hinshaw, D.B., Lodhi, I.J., Hurley, L.L, Atkins, K.B., Dabrowska, M.I., 1999. Activation of poly [ADP-Ribose] polymerase in endothelial cells and keratinocytes: role in an in vitro model of sulfur mustard-mediated vesication. Toxicol. Appl. Pharmacol. in vitro model

Husain, K., Dube, S.N., Sugendran, K., Singh, R., Das Gupta, S., Somani, S.M., 1996. Effect of topically applied sulphur mustard on antioxidant enzymes in blood cells and body tissues of rats. J. Appl. Toxicol. 16, 245-248.

Jafari, M., 2007. Dose- and time-dependent effects of sulfur mustard on antioxidan system in liver and brain of rat. Toxicology 231, 30-39.

Janouchek, B., Horakova, M., Bartak, P., 1987. Treatment of psoriasis with dichlorodiethyl sulfide. Phlebologie 40, 171-175.

Kehe, K., Raithel, K., Kreppel, H., Jochum, M., Worek, F, Thiermann, H., 2007. Inhibition of poly(ADP-ribose) polymerase (PARP) influences the mode of sulfur mustard (SM)-induced cell death in HaCaT cells. Arch. Toxicol. 82, 461-470.

Kehe, K., Szinicz, L, 2005. Medical aspects of sulphur mustard poisoning. Toxicology 214, 198-209.

Kumar, O., Sugendran, K., Vijayaraghavan, R., 2001. Protective effect of various antioxidants on the toxicity of sulphur mustard administered to mice by inhalation or percutaneous routes. Chem. Biol. Interact. 134, 1 12.

Lodhi, L.]., Sweeney, J.F, Clift, R.E., Hinshaw, D.B., 2001. Nuclear dependence of sulfur mustard-mediated cell death. Toxicol. Appl. Pharmacol. 170, 69-77.

Ludlum, D.B., Austin-Ritchie, P., Hagopian, M.. Niu, T.O., Yu, D. 1994, Detection of sulfur mustard-induced DNA modifications. Chem. Biol. Interact. 91, 39-49.

Ludlum, D.B., Papirmeister, B., 1986. DNA modification by sulfur mustards and nitrosoureas and repair of these lesions. Basic Life Sci. 38, 119-125.

Meier, H.L., 1996. The time-dependent effect of $2,2^{\prime}$-dichlorodiethyl sulfide (sulfur mustard HD 1,1'-thiobis [2-chloroethane]) on the lymphocyte viability and the kinetics of protection by poly(ADP-ribose) polymerase inhibitors. Cell Biol Toxicol. 12, 147-153.

Meier, H.L., Gross, C.L., Papirmeister, B., 1987. 2,2'-Dichlorodiethyl sulfide (sulfur mustard) decreases NAD+ levels in human leukocytes. Toxicol. Lett. 39, 109-122

Meier, H.L...Johnson, J.B., 1992. The determination and prevention of cytotoxic effect induced in human lymphocytes by the alkylating agent $2,2^{\prime}$-dichlorodiethyl sulfide (sulfur mustard. HD). Toxicol. Appl. Pharmacol. 113, 234-239.

Meier, H.L. Millard, C., Moser, J., 2000. Poly(ADP-ribose) polymerase inhibitors regulate the mechanism of sulfur mustard-initiated cell death in human lymphocytes. J. Appl, Toxicol. 20 (Suppl. 1), S93-S100.

Mol, M.A., van de Ruit, A.M., Kluivers, A.W., 1989. NAD+ levels and glucose uptake of cultured human epidermal cells exposed to sulfur mustard. Toxicol. Appl. Pharmacol. 98, 159-165.

Mol, M.A., van der Schans, G.P., Lohman, P.H., 1993. Quantification of sulfur mustardinduced DNA interstrand cross-links and single-strand breaks in cultured human epidermal keratinocytes. Mutat. Res. 294, 235-245.

Pant, S.C., Vijayaraghavan, R., Kannan, G.M., Ganesan, K., 2000. Sulphur mustard induced oxidative stress and its prevention by sodium 2,3-dimercapto propane sulphonic acid (DMPS) in mice. Biomed. Environ. Sci. 13, 225-232

Papirmeister, B., Gross, C.L., Meier, H.L., Petrali, J.P., Johnson, J.B., 1985. Molecular basis for mustard-induced vesication. Fundam. Appl. Toxicol. 5, S134-S149.

Paromov, V., Suntres, Z., Smith, M., Stone, W.L., 2007. Sulfur mustard toxicity following dermal exposure: role of oxidative stress, and antioxidant therapy. J. Burns Wounds 7, e7.

Patel, P.S., 2006. Overcoming the force and power of immunity: a history of immunosuppression in kidney transplantation. J. Nephrol. 19 (Suppl. 10), S137S143.

Pleschke, J.M., Kleczkowska, H.E., Strohm, M., Althaus, F.R., 2000. Poly(ADP-ribose) binds to specific domains in DNA damage checkpoint proteins, J. Biol. Chem. 275 , 40974-40980.

Rebholz, B., Kehe, K., Ruzicka, T, Rupec, R.A, 2008. Role of NF-kappaB/RelA and MAPK pathways in keratinocytes in response to sulfur mustard. J. Invest. Dermatol. 128, $1626-1632$.

Rosidi, B., Wang, M., Wu, W., Sharma, A., Wang, H., lliakis, G., 2008. Histone H1 functions as a stimulatory factor in backup pathways of NHE]. Nucleic Acids Res. $36,1610-1623$.

Ruff, A.L., Dillman, J.F, 2007. Signaling molecules in sulfur mustard-induced cutaneous injury. Eplasty 8, e2

Steinritz, D. Emmler, J. Hintz, M., Worek, F, Kreppel, H., Szinicz, L, Kehe, K., 2007 Apoptosis in sulfur mustard treated A549 cell cultures. Life Sci. 80, 2199-2201.

Thomas, H.D., Calabrese, C.R., Batey, M.A., Canan, S., Hostomsky, Z., Kyle, S., Maegley, K.A., Newell, D.R., Skalitzky, D., Wang, L.Z., Webber, S.E., Curtin, N.J., 2007. Pre- 
clinical selection of a novel poly(ADP-ribose) polymerase inhibitor for clinical trial. Mol. Cancer Ther. 6, 945-956.

Vijayaraghavan, R., Sugendran, K., Pant, S.C., Husain, K., Malhotra, R.C., 1991. Dermal intoxication of mice with bis(2-chloroethyl)sulphide and the protective effect of flavonoids. Toxicology $69,35-42$

Wu, W. Wang, M., Singh, S.K., Mussfeldt, T., lliakis, G., 2008, Repair of radiation induced DNA double strand breaks by backup NHEJ is enhanced in G2. DNA Repair (Amst.) 7. 329-338.
Yourick, J.J., Clark, C.R., Mitcheltree, L.W., 1991. Niacinamide pretreatment reduces microvesicle formation in hairless guinea pigs cutaneously exposed to sulfu mustard. Fundam. Appl. Toxicol, 17, 533-542.

Zaremba, T., Curtin, N.J., 2007. PARP inhibitor development for systemic cancer targeting. Anticancer Agents Med. Chem. 7. 515-523.

Zhang, Z., Riviere,J.E., Monteiro-Riviere, N.A., 1995. Evaluation of protective effects of sodium thiosulfate, cysteine, niacinamide and indomethacin on sulfur mustardtreated isolated perfused porcine skin. Chem. Biol. Interact. 96, 249-262. 